\title{
Comment
}

\section{Socialization of scientific and technological research: further comments}

\begin{abstract}
Research systems are increasingly required to be more practically oriented and to address issues which appear more promising in economic and social results, with special reference to trans-disciplinary research fields, such as nanotechnology or ICTs; policy makers show a sharp tendency to establish research priorities and to drive research systems; universities and research institutions are asked to be more transparent and open to dialogue with social actors on contents, impacts, ethical implications and practical applications of scientific and technological research. These transformations affecting both the ways in which science and technology are produced and their relationships with society pose new challenges to European research. All the aspects of research activities are concerned, including the life of the research groups, the approaches to scientific evaluation, the development of European research policies and the interaction between researchers with their social environment. Continuing a reflection started in the last issue of JCOM, Luisa Prista, Evanthia Kalpazidou-Schmidt, Brigida Blasi, Sandra Romagnosi and Miguel Martinez López offered their contribution in identifying some of the key implications and risks which these changes are bringing about, mainly in the perspective of the construction of the European Research Area.
\end{abstract}

In the last issue, JCOM hosted some comments revolving around the changing relationships between science and society, viewed through the conceptual lens of the socialization of scientific and technological research.

This notion focuses on the increasing difficulties met by science - a typical institution of modernity - to adapt itself to a global network-shaped, multi-centric "post-modern" society and to manage the social dynamics which are today massively embodied in scientific and technological research.

The turn from an "academic" to a "post-academic" science reflects this broader change. Research systems are increasingly required to be more practically oriented and to address issues which appear more promising in economic and social results, with special reference to trans-disciplinary research fields, such as nanotechnology or ICTs; policy makers show a sharp tendency to establish research priorities and to drive research systems; universities and research institutions are asked to be more transparent and open to dialogue with social actors on contents, impacts, ethical implications and practical applications of scientific and technological research.

However, these transformations are occurring in a fragmented way, with considerable phase displacements, contradictions, and gaps, involving both the organization of science production and the relations between science and society. Moreover, the still dominant scientific culture and the most widespread representations of science seem not to be able to manage and drive these changes.

All that results in a decrease in science and technology socialization, that is in the level of embeddedness of scientific and technological research in society, with consequences both on research quality and on society.

The new comments published on this issues offer some new foods for thought on these themes.

In her article, Luisa Prista dwells upon the action carried out by the European Commission DG Research to support science and technology socialization, which is mainly pivoted on the "Science in Society" initiative, an autonomous program included in the Seventh Framework Program of Research, to address a profound change in the public's attitude towards science. While last century was marked by a widespread enthusiasm about scientific progress, in this century science is increasingly asked to give solutions to global challenges (such as climate change, mobility, or pandemic flues), which often trigger conflicts and create discontent and distrust towards science. Hence the need to favor a higher embeddedness of science in society, addressing a wide range of issues, such as promoting young people's interest in science, closing the gender gaps in science, promoting debates and reflection on ethical issues in the governance of research, promoting a broader and open role of the universities or developing public engagement in science and 
science communication. However, the road to make is not easy, the direction to take is not unique and the way is not linear.

The Evanthia Kalpazidou-Schmidt's comment focuses on the pivotal role in socializing science and technology played by evaluation in a European perspective. Evaluation provides feedback into the ongoing process of policy making and governance and may support it or suggest changes, allowing the activation of learning processes within the European Research Area (ERA). However, according to the author, the policy of integrating science and technology in the ERA generates new challenges for evaluation, mainly in terms of creating synergies of the plethora of initiatives and the interplay between the instruments of European science and technology policy. In this framework, three points deserve particular attention: the establishment of a more coordinated evaluation system in Europe; the development of a more integrated approach to evaluation; the strengthening of the linkages between the national and European level.

Science and technology socialization perspective could be also helpful in grasping the main research dynamics at the level of micro-analysis. In their article, on the basis of an experimentation carried out at the University of Tor Vergata in Rome, Brigida Blasi and Sandra Romagnosi reflect about this aspect, concentrating the attention on research groups, which represent the smallest organizational unit involved with the research process. The authors offer a picture of the profound changes presently affecting the life of the research groups, such as the influence of industrial models on researchers' training, the redrawing of the disciplinary boundaries and research methods or the changing interests of public research. Overall, these transformations reinforce the collective nature of scientific work, which is often managed with great difficulty, both by individual researchers and at the institutional level. Many scientific systems do not keep pace with organizational and professional innovation, not adapt to the contemporary real needs and eventually not reach the steady state. The creation and the maintenance over the time of a research group is therefore to be viewed as an event not to be taken for granted but as a process to be developed step by step and continuously at risk of interruption.

Miguel Martinez López, in his article, analyses the notion of "scientific mediation" as a tool for shedding light on the science and technology socialization processes. In particular, this notion helps to draw the attention on the often complex fabric of social relations that scientists establish with their social environment, which involve a vast array of players, different social ties, channels and codes of communication as well as specific persons acting as mediators, either professionals or not. As the author points out, this set of interactions are not to be viewed as a distorting factors. Science and technology cannot exist in a social vacuum. It is the society to provide them with resources, demands, values, information and people science and technology need for developing. Scientists need the influence of other scientists as well as the support of managers, friends, relatives, students, assistants, journalists, and other actors. Studying these ties is a necessary step in order to understand the actual social networks in which real scientists are located and to grasp how the real research works. 